\title{
AN EXTENDED HAND MOVEMENT MODEL FOR HAPTIC-BASED REMOTE OPERATION OF INFRASTRUCTURE MAINTENANCE ROBOTS
}

\author{
Pholchai Chotiprayanakul*, Dikai Liu, and Gamini Dissanayake \\ Centre for Autonomous Systems, Faculty of Engineering, University of Technology, Sydney, Australia \\ * Corresponding author (pchotipr, dkliu, gdissa@eng.uts.edu.au)
}

\begin{abstract}
When human operator is in the control loop of a robotic system, which operates in unstructured, complex and dynamic environments such as those in construction and steel bridge maintenance, human factors will have significant effect on the operational performance and control of the robot. This paper aims to investigate this effect and develop efficient methods for operational performance improvement. An eXtended Hand Movement (XHM) model for eye-guided hand movement is developed with the aim of providing natural and comfortable interaction between a human operator and a robot. A haptic force-speed-accuracy control method is then designed based on the XHM model and implemented in a steel bridge maintenance robotic system. An experiment is conducted to verify the model and control method. Experimental results demonstrated significant improvement in control accuracy and operational performance in a steel bridge maintenance robotic system.
\end{abstract}

Keyword: Human-Robot Interaction, Haptic Interface, Human Factor, Steel Bridge Maintenance Robot

\section{INTRODUCTION}

Industrial robots have been widely used to perform welldefined repetitive tasks in carefully constructed simple environments such as manufacturing factories. The future of industrial robots is to operate in complex, unstructured and unknown (or partially known) dynamic environments such as those in construction and steel bridge maintenance, to assist human workers in undertaking hazardous tasks. Autonomous operation of industrial robots in such environments is ideal, but there are a lot of challenges that need to be addressed. Semi-autonomous or manual operation with human interaction is a practical solution because it utilises human intelligence and experience combined with the power and accuracy of an industrial robot. Therefore, haptic-based human-robot interaction provides an enabling methodology. However, there are still many challenging issues which need to be solved.

When human operator is in the control loop of a robotic system, human factors will have significant effect on the performance of haptic-based human interactive operation. This paper aims to investigate this effect and an efficient method for operational performance improvement. An
eXtended Hand Movement (XHM) model for eye-guided hand movement is investigated in this paper with the aim of providing natural and comfortable interaction between a human operator and a robot, and improving the operational performance. The model is studied for increasing the speed of the robot manipulator while maintaining the control accuracy. This model is then applied into a steel bridge maintenance robotic system [1][21].

Fig. 1 shows the robotic system developed for sandblasting operation in steel bridge maintenance. It consists of a robot arm affixed with sandblasting equipment and placed on a mobile platform. A haptic device with force feedback is used to operate the robot in a complex environment. A virtual spring method [2] is used as the haptic device and robot arm interface for guiding the robot arm to target points. Attractive force (AF) method [3][4] and three dimensional virtual force field (3D-VF') method [5] are also used for target position control and real time collision avoidance. When a human operator is in the control loop of the robot arm by means of the haptic device, for example sandblasting path planning, end-effector (or sandblasting nozzle) motion control and robot collision avoidance, the 
movement of the end-effector (the whole robot arm as well) will be significantly affected by hand movement of the operator.

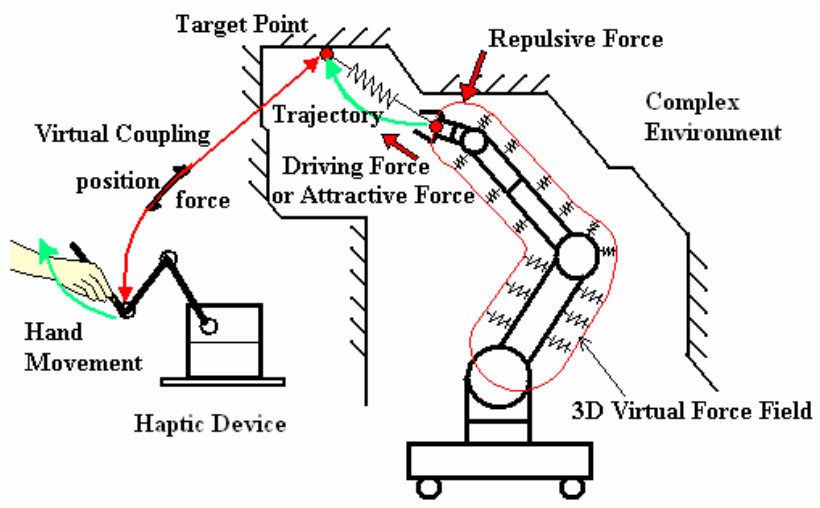

Fig. 1 Haptic-based sandblasting robot arm

The popular model of human hand movement is Fitts's law [8], which describes human psychomotor behaviour. Fitts's law has been used to predict movement time and indicate the difficulty of using computer-input devices. This law has been mathematically formulated upon data gathered from experiments using a single dimension movement testing method and a circular testing method. In 1997, Accot and Zhai [7] presented a well-known extension to the Steering law of human hand movement. Accot and Zhai derived an index of difficulty for Fitts's law in an integrated form, which can be used to test movements along a 2D tunnel path. Accot-Zhai's Steering law [7][13] is now used to evaluate GUI design in computer applications, operating systems and computer-console games. The two most common design guidelines for software and hardware interaction are ISO 9241-920:2009-3: Ergonomic of Human System Interaction for Tactile and Haptic Hardware and Software Interaction, and ISO 9241-9: Virtual Display Terminals (VDTs) - requirements of nonkeyboard input device [9]. However, the Fitts' law and Steering law cannot be directly used to measure performance of the human-robot interaction in operation of the haptic-based sandblasting robot arm. For sandblasting process, operational performance is measured based on speed and accuracy of blasting spot on surface target [15]. Thus, the Fitts' law and Steering law need to be modified in order to integrate the speed and accuracy of the blasting spot into these laws.
In haptic-based operation, the haptic force generated by a haptic device resists the movement of an operator's hand. The haptic force therefore affects the operator's hand movement, and consequently affects the pointing accuracy of the manipulator end-effector (sandblasting spot) on the task plane. The research presented in [2][12] only provides a relationship between feedback force and pointing errors. Therefore, the relationship between the pointing accuracy, the haptic force, and speed of the end-effector of manipulator need to be considered in human hand movement model.

\section{EXTENDED HUMAN HAND MOVEMENT MODEL}

From Accot-Zhai's Steering law [7][13] included the effect of control scale [12] as shown in equation 1, the movement time $(M T)$ is defined by the travelling distance $(s)$, path width $(\phi)$, the control scale $\left(G_{x}\right)$, the start/stop time $(a)$, and the coefficient of movement speed $(b)$. Start/stop time $(a)$ is the time that an operator spends for planning their movement.

$M T=a+b \frac{s}{G_{x} \phi}$

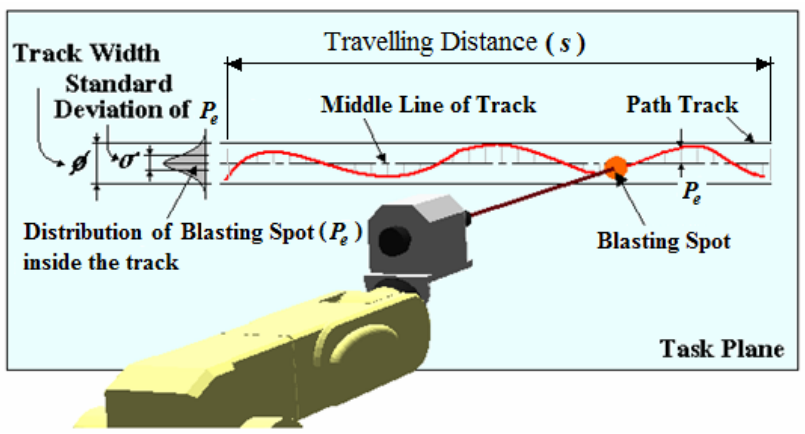

Fig. 2 Distribution of the sandblasting spot $\left(P_{e}\right)$ inside a path track

When the cursor of a pointing device is manually controlled and moved through a predefined path-track, the distribution of the cursor positions inside the target area is a normal distribution about a mean ( $\mu$ ) which is approximated as the middle line of the path-track. The standard deviation $(\sigma)$ can be used to measure the pointing accuracy. $Z_{a c} \sigma$ defines the pointing accuracy, where $Z_{a c}$ is 
the coefficient of standard deviation. The models of human hand movement in Steering law can be modified as:

$$
M T=a+b \frac{s}{G_{x}\left(\phi-Z_{a c} \sigma\right)}
$$

For this modification, width of the path-track needs to be greater than the pointing accuracy $\left(\phi-Z_{a c} \sigma>0\right)$.

\subsection{Pointing Accuracy}

When a haptic device with force feedback is used as a pointing device, the distribution of the cursor position, which is represented by a standard deviation $(\sigma)$, is affected by the power generated by the haptic device, which is product of the haptic force $\left(F_{h}\right)$ and the speed of the operator's hand (i.e. the haptic cursor speed; $v_{a}$ ). In this paper, $\sigma$ is therefore assumed as a function of the haptic force and hand movement speed, $F_{h}$ and $v_{a}$ :

$\sigma=k_{p w} F_{h} v_{a}+\sigma_{0}$

where $\sigma_{0}$ is a constant and $k_{p w}$ is a coefficient of power. The hand movement model in the Steering law as shown in equation 1 can be rewritten as

$$
M T=a+b \frac{s}{G_{x}\left(\phi-Z_{a c}\left(k_{p w} F_{h} v_{a}+\sigma_{0}\right)\right)}
$$

\subsection{Extended Hand Movement Model}

From the hand movement model of the Steering law in equation 4, when start/stop time $(a)$ does not directly depend on the hand movement of an operator, MT- $a$ will be the time that the operator spends for moving the hand. Thus, the speed of hand movement $\left(v_{a}\right)$ can be defined as:

$$
v_{a}=\frac{s}{M T-a}=\frac{G_{x}\left(\phi-Z_{a c}\left(k_{p w} F_{h} v_{a}+\sigma_{0}\right)\right)}{b}
$$

The extended hand movement model (XHM) is defined by

$v_{a}=\frac{\phi-Z_{a c} \sigma_{0}}{b G_{x}^{-1}-Z_{a c} k_{p w} F_{h}}$

From experimental results presented in [20], the coefficient of movement $(b)$ is obtained as $0.1958 \mathrm{sec}, k_{p w}$ is equal to $0.0077 \mathrm{~m} / \mathrm{W}$, and $\sigma_{0}$ is $0.00057 \mathrm{~m}$. Fig. 3 shows the extended hand movement model with different accuracy requirements at $\phi=12.7 \mathrm{~mm}$ and $G_{x}$ is set to 1 .

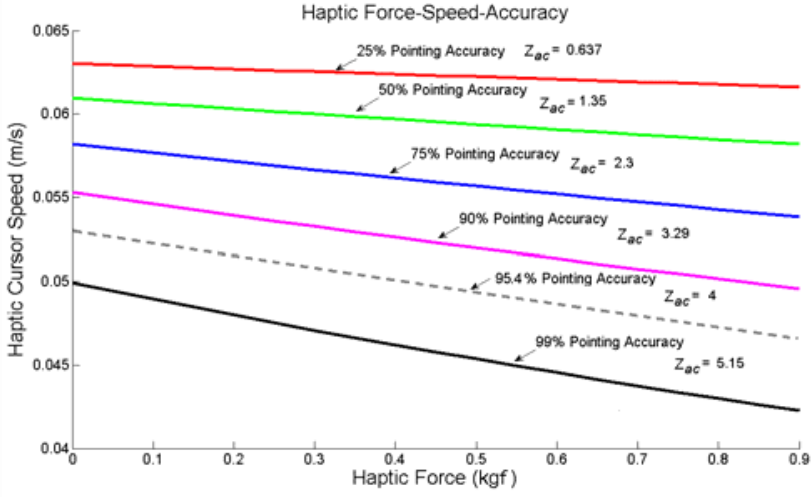

Fig. 3 Haptic force-cursor speed graph with different pointing accuracy, $\phi=12.7 \mathrm{~mm}$.

From Fig. 3, if the required accuracy of movement is low (e.g., 25\%), the movement speed can be high. At the same level of accuracy, if a big force is needed, the movement speed is reduced. On the other hand, if the required accuracy of movement is high (e.g., 99\%), the hand movement needs to be slow in order to achieve the accuracy. When an operator has to apply a force (including a haptic force) to move an object (which is similar to applying a haptic force to move a cursor of a haptic device), the operator normally moves slowly in order to have high accurate movement. If the required force to move an object is small, the operator normally tries to move faster until the movement is found to be inaccurate, and the operator then reduces the speed of the movement.

\subsection{Haptic Force-Speed Control Method}

In order to improve the haptic-based operation, the extended hand movement (XHM) model has been integrated into a Haptic Force-Speed Control (HFSC) method. Fig. 4 shows two control diagrams: the control diagram of the haptic-based interaction system without the HFSC method (Fig. 4a) and the control diagram with the HFSC method (Fig. 4b). 
$\mathrm{F}_{\text {op }}$
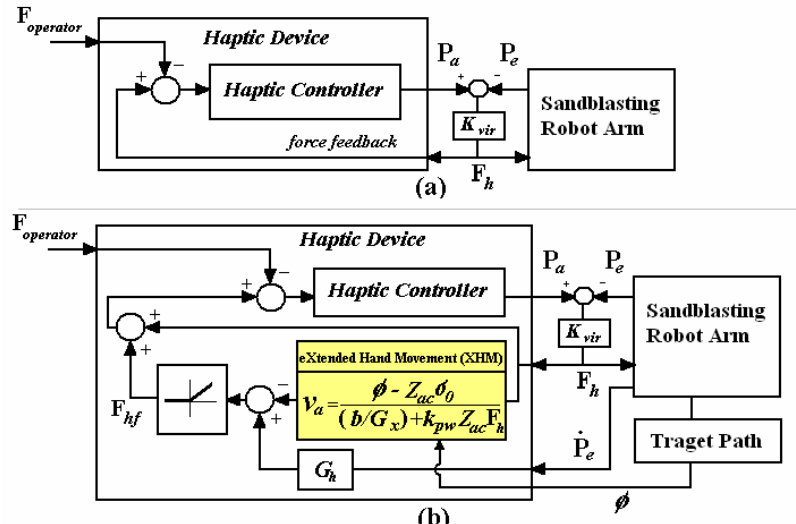

(b)

Fig. 4 Control diagrams of haptic based interaction: (a) without HFSC method, (b) with HFSC method

As shown in Fig. 4b, in this HFSC method, the XHM model is used to calculate a haptic speed $\left(v_{a}\right)$ from an instant haptic force $\mathrm{F}_{\mathrm{h}}$. The speed $v_{a}$ calculated from the XHM model is compared with the actual haptic cursor speed $G_{x} \dot{P}_{e}$. If $G_{x} \dot{P}_{e}$ is greater than $v_{a}$, the speed difference $\left(G_{x} \dot{P}_{e}-v_{a}\right.$ ) is amplified and converted to force $\left(F_{h f}\right)$ that is added into the haptic force $F_{\mathrm{h}}$. Because of this force addition, the movement of the operator's hand is subject to more resistance than usual. As a result, the speed of the operator's hand is reduced and therefore the point accuracy is increased. When the haptic force and the speed of the haptic cursor are controlled by the HFSC method, the control accuracy is increased to the desired accuracy as set by $Z_{a c}$ in the XHM model.

To verify this HFSC control method, an experimental test is conducted with the sandblasting robot. The robot endeffector is required to follow a defined path on the surface of a wall. In order to reduce the redundancy in robot pose and joint force calculation, only the first three joints (Joints 1,2 , and 3) of the manipulator are controlled and the other three joints are set to rigid. The haptic force, which is generated by the virtual spring-based haptic force generation method [2][3], is measured by a 6DOF force sensor on the haptic device handle. The pointing accuracy and the operating speed are measured and used to analyse the improvement in performance.

\section{EXPERIMENT}

In the experimental test, the participant controls the endeffector of the manipulator (i.e. the blasting spot) to move clockwise inside the circular path on the surface of a wall by using the Novint ${ }^{\circledR}$ haptic device. The participant is required to move the blasting spot as close as possible to the middle line of the track and to keep it inside the track boundaries. The test period is one minute and this test is repeated for five times. Figure 5 shows the setup of this experimental test. The circular path track is set up on a vertical wall in front of the manipulator. The medium radius $\left(r_{b}\right)$ of the track is $375 \mathrm{~mm}$ and four track widths are used: $20 \mathrm{~mm}, 40 \mathrm{~mm}, 60 \mathrm{~mm}$ and $80 \mathrm{~mm}$.

In this test, the resistant force in each of the three joints is assumed to be viscous damping force. When operating in a real 3D environment, repulsive forces generated from the $3 \mathrm{D}-\mathrm{VF}^{2}$ method [20] based on distance from the robot arm to obstacles are a kind of resistant force that is used for collision avoidance. All the damping forces in the three joints will affect the speed and displacement of the endeffector according to the dynamic model of the robot as presented in [3][4], and will consequently affect the haptic force according to the haptic force generation method.

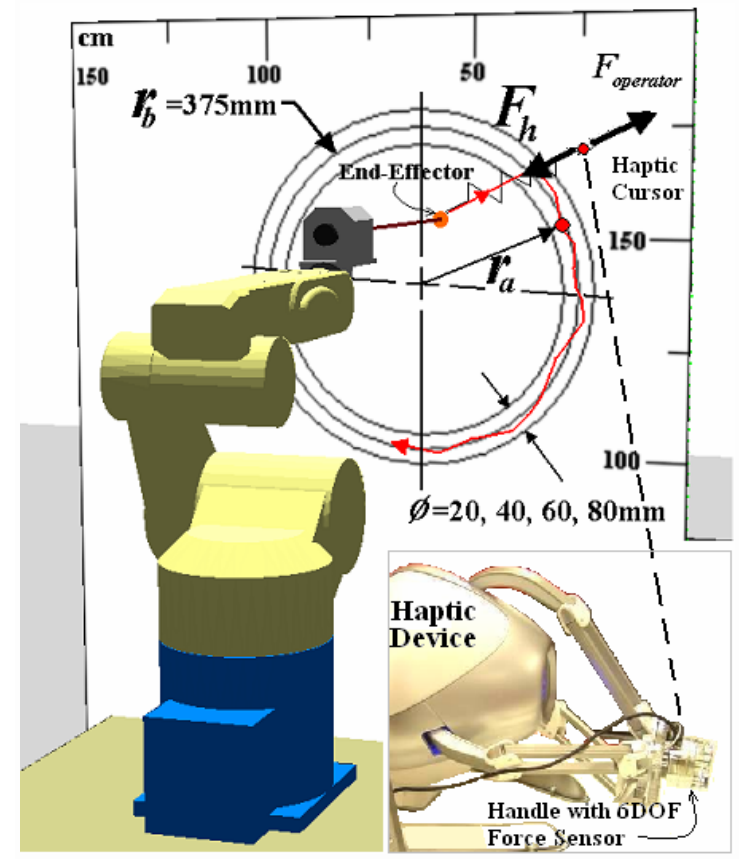

Fig. 5 Test setup 


\section{RESULT}

The position of the end-effector of the manipulator is represented by $r_{a}$ and the speed of the end-effector of the manipulator is represented by $\dot{P}_{e}$ as shown in Fig. 5. The position and the speed of the end-effector and the haptic force are recorded during the test. Fig. 6 shows the recorded data of a participant in one of five repeats. In each figure, for example Fig.6(a), the graph on the left-hand side shows the haptic force-end-effector speed distribution. The top-right graph presents the end-effector position, $r_{a}$, obtained in the test without the HFSC method. The bottomright graph presents $r_{a}$ in the test with the HFSC method. The standard deviation ( $\sigma$ ) of the end-effector position $r_{a}$ is calculated and shown in the two right-hand graphs.

It can be seen from Fig. 6 that the pointing accuracy in the haptic-based operation is increased (i.e. reduction of $\sigma$ ) when the path-track width of the desired task is getting wider, which enables the operator to control the robot more easily than in the narrow path-track width. When the HFSC method is used, the resistant force is increased by the XHM model. Consequently, the speed of the blasting spot is limited under the desired pointing accuracy. Therefore, the HFSC method helps operators to operate more accurately than a system that does not use the HFSC method.

Table 1 shows the average standard deviations $(\sigma)$ of endeffector position, $r_{a}$, in the five repeats. It can be seen from the table that the standard deviation with the HFSC method (4th row) is lower than the standard deviation without the HFSC method (3rd row); therefore, the pointing accuracy is improved with the HFSC method.

Table 1. Average standard deviations $(\sigma ; \mathrm{mm})$ of 5 repeats with different path-track widths

\begin{tabular}{|l|c|c|c|c|}
\hline & \multicolumn{4}{|c|}{ Standard deviation $(\sigma ; \mathrm{mm})$} \\
\hline Track width (mm) & 20 & 40 & 60 & 80 \\
\hline without HFSC & 11.3 & 12.8 & 16.3 & 16.2 \\
\hline with HSFC & 6.5 & 5.4 & 8.9 & 9.7 \\
\hline
\end{tabular}

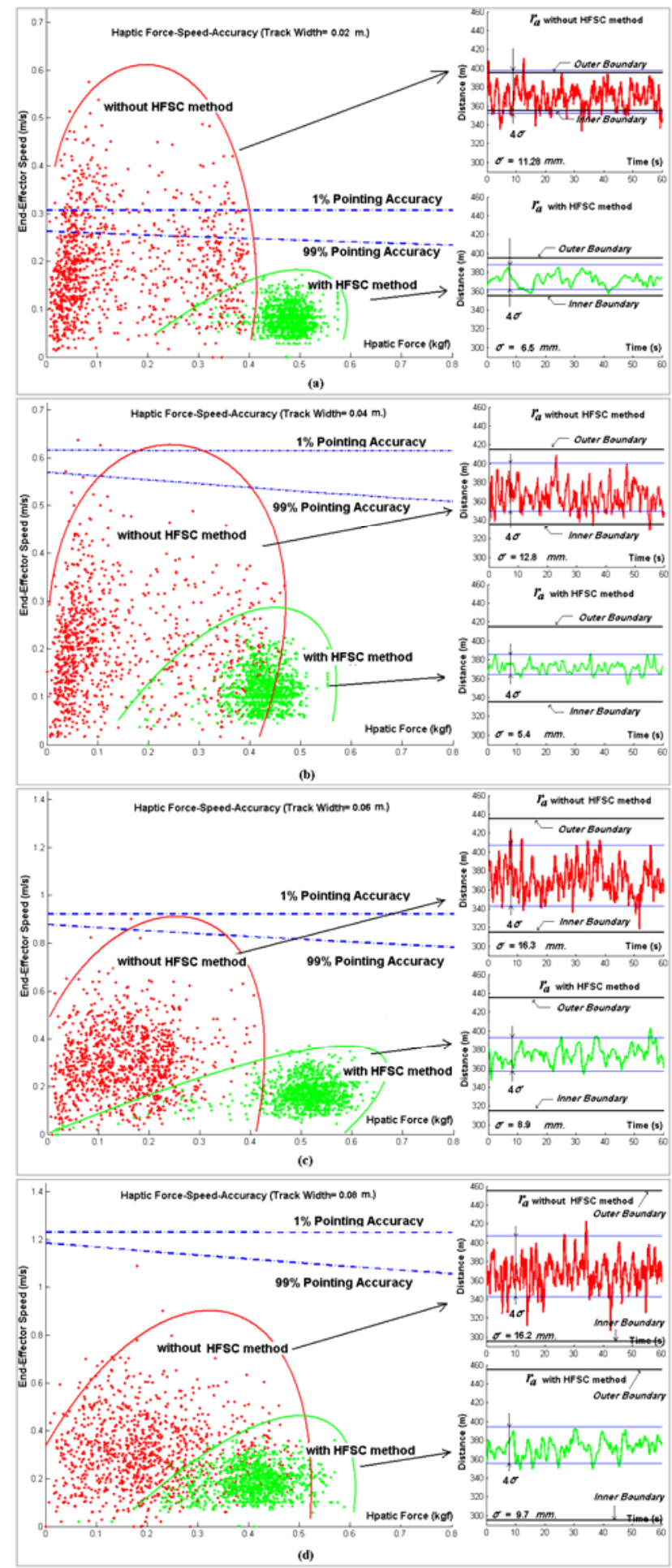

Fig. 6 Experimental results with different track width: (a) 20mm, (b) 40mm, (c) 60mm, and (d) $80 \mathrm{~mm}$.

\section{CONCLUSION}

This paper has investigated the effect of human hand movement on haptic-based robot operation and an HFSC method to improve the control accuracy. An eXtended 
Hand Movement (XHM) model has been developed to present human hand movement as function of haptic force and pointing accuracy. To evaluate this XHM model, a control method, i.e. the Haptic Force-Speed Control (HFSC) method, has been developed and integrated into a sandblasting robot control system. An experimental test has been conducted to verify this HFSC method. Experiment results show that the HFSC method can significantly improve the control accuracy of the haptic-based robot operation.

\section{REFERENCES}

[1] D. K. Liu, G. Dissayanake, P. B. Manamperi, P. A. Brooks, G. Fang, G. Paul, S. Webb, N. Kirchner, P, Chotiprayanakul, N. M. Kwok and T. R. Ren, 2008, ‘A Robotic System for Steel Bridge Maintenance: Research Challenges and System Design', paper presented at the 2008 Australasian Conference on Robotics and Automation, 3-5 December 2008, Canberra, Australia.

[2] J. Park and O. Khatib, 2006, 'A Haptic Teleoperation Approach Based on Contact Force Control', International Journal of Robotics Research, vol. 25, no. 5-6, pp. 575-591.

[3] P. Chotiprayanakul, D. Wang, N. M. Kwok and D.K. Liu, 2008, ‘A Haptic Base Human Robot Interaction Approach for Robotic Grit Blasting', Proceedings of the 25th International Symposium on Automation and Robotics in Construction (ISARC’2008), pp. 148-154.

[4] P. Chotiprayanakul and D. K. Liu, 2009, 'Workspace Mapping and Force Control for Small Haptic Device Based Robot Teleoperation', Proceedings of the 2009 IEEE International Conference on Information and Automation (ICIA’2009), pp. 1613-1618.

[5] P. Chotiprayanakul, D. K. Liu, D. Wang and G. Dissanayake, 2007, ‘A 3-Dimensional Force Field Method for Robot Collision Avoidance in Complex Environments', Proceedings of the 24th International Symposium on Automation and Robotics in Construction (ISARC’2007), pp. 139-145.

[6] J. Accot and S. Zhai, 2001, 'Scale Effects in Steering Law Tasks', Proceedings of the ACM Computer
Human Interface Conference, Spatial Interest Group on Computer Human Interaction (SIGCHI), vol. 3, no. 1, pp. 1-8.

[7] J. Accot and S. Zhai, 1997, 'Beyond Fitts' Law: Models for Trajectory-Based HCI Tasks', Proceedings of the ACM Computer Human Interface Conference, Spatial Interest Group on Computer Human Interaction (SIGCHI), pp. 295-302.

[8] P. M. Fitt, 1954, 'The Information Capacity of the Human Motor System in Controlling the Amplitude of Movement', Journal of Experimental Psychology, vol. 47, no. 6, pp. 381-391.

[9] D. Natapov, S. J. Castellucci and I. S. MacKenzie, 2009, 'ISO 9241-9: Evaluation of Video Game Controllers', Proceedings of the ACM Graphic Interface Conference 2009, pp. 223-230.

[10] R. J. Teather, A. Pavlovych and W. Stuerzlinger, 2009, 'Effect of Latency and Spatial Jitter on 2D and 3D Pointing', Proceedings of the IEEE Virtual Reality Conference 2009, pp 229-230.

[11]X. Ren, K.o Shinomori and Y. Kimura, 2006, 'S-H Model and Its Application in Human Interface Design', Kochi University of Technology Research Bulletin, vol. 3, no. 1, pp. 55-64.

[12] G. Casiez, D. Vogel, R. Balakrishnan, and A. Cockburn, 2008, 'The Impact of Control-Display Gain in User Performance in Pointing Tasks', International Journal of Human-Computer Interaction, vol. 23, pp. 215-250

[13] J. Accot and S. Zhai, 2003, 'Refining Fitts' Law Models for Bivariate Pointing', Proceedings of the International Conference on Computer-Human Interaction 2003, vol. 5, no. 1, pp. 193-200.

[14] G. S. Lee and B. Thuraisingham, 2008, 'Differences in Fitts’ Law Task Performance Based on Environment Scaling', Haptics: Perception, Devices and Scenarios: 6th International Conference EuroHaptic 2008, Springer-Verlag, Berlin, pp. 295-300.

[15] Mikell P. Groover, 2007, ‘Automation, Production Systems, and Computer-Integrated Manufacturing System’, Prentice Hall, pp.49-68.

[16] Richard C. Simpson, 2009, 'Using the Speed- 
Accuracy Operating Characteristic to Visualize Performance with Pointing Device', Journal of The Open Rehabilitation, vol.2, pp. 58-63.

[17] J. Z. Liu, R. W. Brown and G. H. Yue, 2002, ‘A Dynamical Model of Muscle Activation, Fatigue, and Recovery', The Biophysical Journal, vol. 82, pp. 23442359.

[18] L. Ma, D. Chablat, F. Bennis, W. Zhang and F. Guillaume, 2008, ‘A New Muscle Fatigue and Recovery Model and Its Ergonomics Application in Human Simulation', Proceedings of IDMME-Virtual Concept 2008, pp. 1-10.

[19] L. M. Munoz and A. Casals, 2009, 'Improving the Human-Robot Interface Through Adaptive Multispace Transformation', IEEE Transactions on Robotics, vol. 25, no. 5, pp. 1208-1213.

[20] P. Chotiprayanakul, 2011, 'Effective Methods for Human-Robot-Environment Interaction by means of Haptic Robotics', PhD thesis, University of Technology, Sydney, submitted Dec 2010.

[21]G. Paul, D.K. Liu, N. Kirchner and G. Dissanayake (2009), “An effective approach to simultaneous mapping and surface-type identification of complex 3d environments”, Journal of Field Robotics, 26 (11-12): pp915-933, November-December 2009 